\title{
'Design of power factor meter using internet of things for power factor improvement, remote monitoring and data logging
}

\author{
Teddy Surya Gunawan ${ }^{1}$, Muhamad Hadzir Anuar ${ }^{2}$, Mira Kartiwi ${ }^{3}$, Zuriati Janin ${ }^{4}$ \\ ${ }^{1,2}$ Department of Electrical and Computer Enginering, International Islamic University Malaysia, Malaysia \\ ${ }^{1}$ School of Electrical Engineering and Telecommunications, University of New South Wales, Australia \\ ${ }^{3}$ Departement of Information Systems, International Islamic University Malaysia, Malaysia \\ ${ }^{4}$ Faculty of Electrical Engineering, Universiti Teknologi MARA, Malaysia
}

\begin{tabular}{l}
\hline \hline Article Info \\
\hline Article history: \\
Received May 5, 2019 \\
Revised Jul 6, 2019 \\
Accepted Jul 20, 2019 \\
\hline
\end{tabular}

\section{Keywords:}

Data logging

IoT

Power factor

Power factor improvement

Remote moniroting

Smart meter

\begin{abstract}
Nowadays, many residential and commercial buildings that used electricity needs to take care the power factor to avoid penalty from the utility companies. A power factor that is close to one provides a good indicator for the overall power quality. Therefore, power factor improvement plays a significant role to reduce electricity consumption and more efficient system operation. In this paper, the design of power factor meter using Internet of Things will be discussed. Voltage and current sensors outputs were interfaced to Arduino, in which the real power and apparent power were calculated to determine the power factor. Results showed the effectiveness of our proposed device in measuring power factor. Moreover, the measured data points were logged in an SD card and can be accessed by computer with Matlab graphical user interface (GUI). In addition, IoT framework analysis for smart meter which can provide power factor improvement, remote monitoring, and data logging was further discussed in this paper.
\end{abstract}

Copyright $@ 2020$ Institute of Advanced Engineering and Science. All rights reserved.

\section{Corresponding Author:}

Teddy Surya Gunawan,

Department of Electrical and Computer Engineering,

Internation Islamic University Malaysia, Malaysia.

Email: tsgunawan@iium.edu.my

\section{INTRODUCTION}

Nowadays electricity is the main power generator that is needed to turn on most of the devices like electronics equipment. Electricity enables us to use various electrical appliances like television, oven, air condition, refrigerator and others. Generally, electric power can be generated using renewable and nonrenewable sources. Most electric companies charge either the highest $\mathrm{kW}$ (real power), or the highest kVA (apparent power), whichever is greater [1]. If the power factor is low, the measured kVA will be significantly higher than the $\mathrm{kW}$. Therefore, improving the power factor through power factor correction, e.g. capacitor bank connected in parallel, will lower the kVA requirement thus lower the electricity bill. Several other advantages of power factor improvement, including increased load carrying capabilities in existing circuits, improved voltage, reduced power losses, and reduced carbon footprint [1].

Many research have been conducted on the development of smart meter as discussed in [2-4]. Due to its usage, smart meters are being developed and installed in many homes, school buildings, universities, and industrial premises around the world [5,6]. The smart meter infrastructure, if used properly, can provide more than just recoding consumption of electricity, such as easier processing of billing, detection of energy losses due to possible fraud, early warning of blackouts, as well as precision real-time pricing schemes [7].

One of the advantages using the digital based power factor meter is that the read data by the device can be stored easily for other applications or analysis $[8,9]$. One type of the analog circuit that used in the power factor measurement system is zero crossing detectors to convert and process the current and voltage 
signals, in which the output will be low if the difference is zero [10]. In [11], the firmware design can be used to calculate and record power consumption data and record five outage events with their interruption time, interruption durations and restoration time if an outage event occurred. Figure 1 shows various samples of power factor/smart meter.
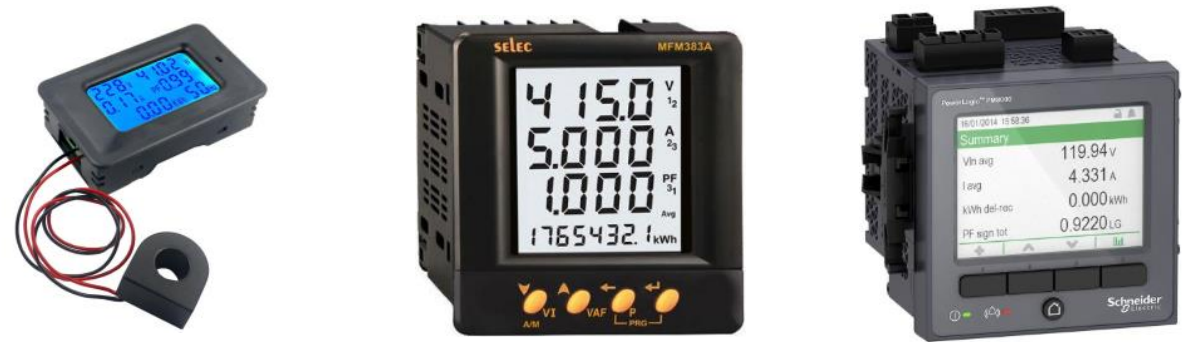

Figure 1. Examples of commercial power factor meters

Although many researches have been conducted on the power meter, but little research has focused on the power factor meter, especially for power factor improvement, remote monitoring, and data logging. In [12], the design of power factor meter was elaborated. The objective of this paper is to improve and extend the previous design by including simulation of power factor improvement, performance evaluation using several devices, remote monitoring, and data logging capability.

\section{POWER MEASUREMENT THEORY}

Average power is the average of instantaneous power over one period as shown in (1), in terms of effective or $R M S$ value. The average power consists of real and imaginary power. If the phase angle between the voltage and current are the same, it implies that the load is purely resistive and all power is being absorbed by the load. If $\theta_{v}-\theta_{i}= \pm 90^{\circ}$, the load is considered as reactive and the average power is being reflected back to the $A C$ (alternating current) source.

$$
\begin{aligned}
& P=V_{R M S} I_{R M S} \angle\left(\theta_{v}-\theta_{i}\right)=V_{R M S} I_{R M S} \cos \left(\theta_{v}-\theta_{i}\right) \\
& S=V_{R M S} \times I_{R M S} \\
& I_{R M S}=\sqrt{\sum_{n=0}^{N-1} \frac{i^{2}(n)}{N}} \\
& V_{R M S}=\sqrt{\sum_{n=0}^{N-1} \frac{v^{2}(n)}{N}}
\end{aligned}
$$

Apparent power $(S)$ is the voltage of an $A C$ system multiplied by all the current that flows into it. It can be computed as the product of $R M S$ voltage and $R M S$ current, as shown in (2) and is expressed in units of volt-amperes (VA). $V_{R M S}$ and $I_{R M S}$ values are calculated using (3) and (4), where $N$ is the number of samples, $i(n)$ and $v(n)$ are the samples of the electrical current and voltage signals.

Real Power $(P)$ is the capacity of the circuit for performing work at a time. This can only be calculated by measuring voltage and current simultaneously and multiplying them, and averaging over time:

$$
P=\frac{1}{N} \sum_{n=0}^{N-1} v(n) \times i(n)
$$

The Power Factor $(P F)$ is calculated as the ratio between Real Power to Apparent Power. It refers to the ability of the electrical systems on the installation to convert electric current into useful workload such as heat, rotation, or light.

$$
P F=\cos (\theta)=\frac{\text { Real Power }(W)}{\text { Apparent Power }(V A)}=\frac{P}{S}
$$


The $P F$ value is ranging from 0 to 1 which represents the load characteristics, including resistive, inductive, or capacitive. Maximum power transfer happens if the power factor equals to $1(\cos \theta=0)$. If the current leads voltage by $-90^{\circ}$ phase, the load is purely capacitive. While if the current lags voltage by $90^{\circ}$ phase, the load is purely inductive. If the power factor is poor, the load will drive more current, which means more energy lost in the electrical distribution system. Most of the load in the electrical system is having inductive characteristic [10]. Therefore, the industry is normally using capacitor bank connected in parallel to improve the power factor [1].

\section{POWER FACTOR CORRECTION}

A high Power Factor index gives the following benefits, including no additional charges in monthly electricity bill as there is no Power Factor Surcharge, extends the lifespan of electrical appliances, reduces electricity wastage for electrical installations at customers' premises, and conserves the environment by lowering fuel usage and helps the country reduce carbon dioxide (CO2) emissions [1]. This section described the related IEC and IEEE standard on power quality, power factor correction unit, and power factor improvement calculation.

\subsection{IEC and IEEE Standard on Power Quality}

The IEC 61000 series [13] is one of the most commonly used references for power quality in Europe. It contains six parts, including general, environment, limits, testing and measurement techniques, installation and mitigation guidelines, and generic standard. Meanwhile, IEEE-Std 519 [14] is the IEEE recommended practices and requirements for harmonic control in electric power systems. It is more comprehensive than IEC 61000-3-2 [15]. IEEE-Std 519 section 7 deals with converter power factor, reactive power compensation, and control of harmonics. In addition, IEEE-Std 1036 [16] covers on the use of $50 \mathrm{~Hz}$ and $60 \mathrm{~Hz}$ shunt power capacitor units or capacitor banks.

\subsection{Power Factor Correction Unit}

Power factor correction (PFC) unit in the form of capacitor banks as shown in Figure 2(a), which act as reactive current generators, primarily consist of six major parts, including incoming isolators, fuses, capacitor switching contactors, capacitors, power factor regulator, and detuned reactors. By providing the reactive current, PFC reduce the total amount of current the system must draw from the main distribution [17], PFC is normally.

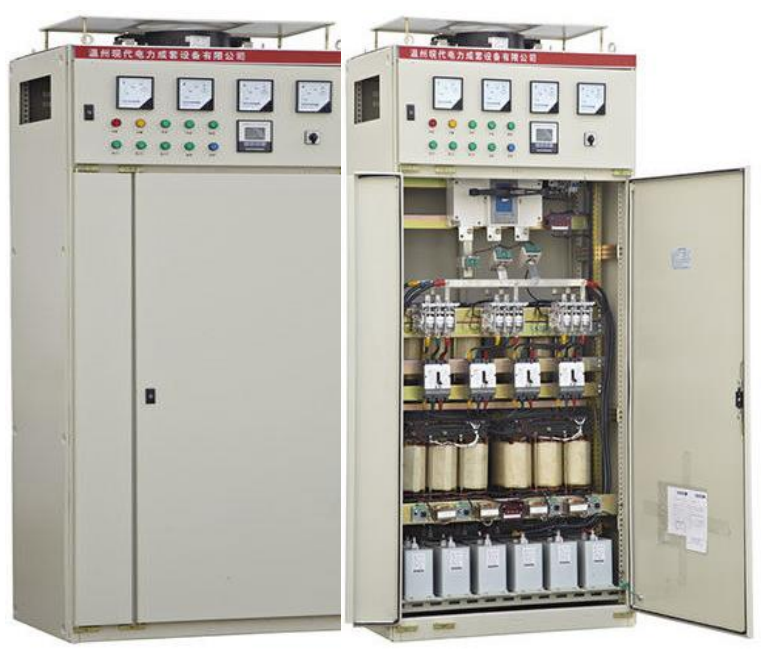

(a) 150kVAR PFC sample unit

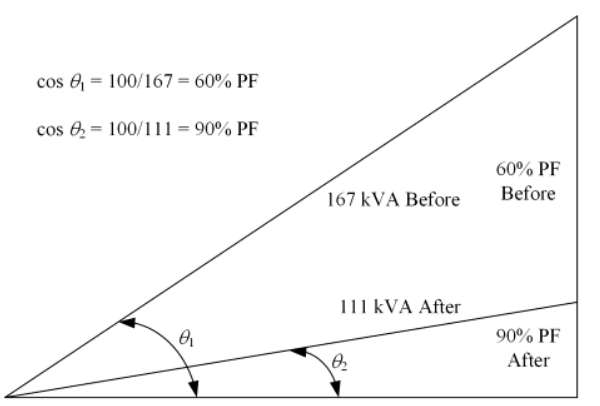

(b) PFC sample calculation

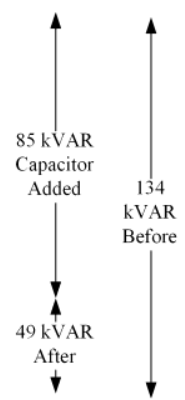

Figure 2. Power factor correction (a) Sample unit, (b) Sample calculation

\subsection{Power Factor Correction Calculation}

The size of the capacitor is calculated as shown in (7) to properly offset inductive load, where $k V A R$ is the PFC capacitor size, $P$ is the real power in $k W, P F_{1}$ is the current power factor, $P F_{2}$ is the targeted power factor [17]. 


$$
k V A R=P \times\left(\tan \left(\cos ^{-1}\left(P F_{1}\right)\right)-\tan \left(\cos ^{-1}\left(P F_{2}\right)\right)\right)=P \times\left(\tan \theta_{1}-\tan \theta_{2}\right)
$$

For example, suppose that we have original $P=100 \mathrm{~kW}$ and $k V A=167$ which produces $P F_{1}=$ $\cos \left(\theta_{1}\right)=\frac{100}{167}=0.6$. If we want the improved power factor to be 0.9 , i.e. $P F_{2}=0.9$, using (7) it requires PFC capacitor size of $k V A R=100 \times\left(\tan \left(\cos ^{-1}(0.6)\right)-\tan \left(\cos ^{-1}(0.9)\right)\right)=85$. Note that, due to the rounding, the new power factor will be $P F=\cos \left(\tan ^{-1}\left(\frac{134-85}{100}\right)\right) \approx 0.9$. Figure 2(b) illustrates this example. The required capacitor in $\mu F$ can then be calculated using $(8)[18,19]$.

$$
C=\frac{k V A R}{2 \pi f V^{2}}
$$

\section{POWER FACTOR METER DESIGN}

In this section, the improvement and extension from [12] on hardware and software design of proposed power factor meter will be presented, including power factor improvement using capacitor banks.

\subsection{Hardware Design}

Figure 3 shows the block diagram of the proposed power factor meter using Arduino. Arduino Uno is the main microcontroller unit which connects to sensors and other output for data logging. For the proper date and time stamp of the data logging, an RTC (real time clock) module is utilized. It features could be further extended using a GPS module which could provide a synchronized date and time, as well as, geo-location, as shown in Figure 2. The output of the power factor measurement could be displayed in an LCD, recorded in an SD card, connected to PC with Matlab using a USB cable for data plotting and further analysis, or sent to a server using WiFi shield [20,21]. The sensors selection, interface circuit, LCD and SD card connection has been discussed in [12].

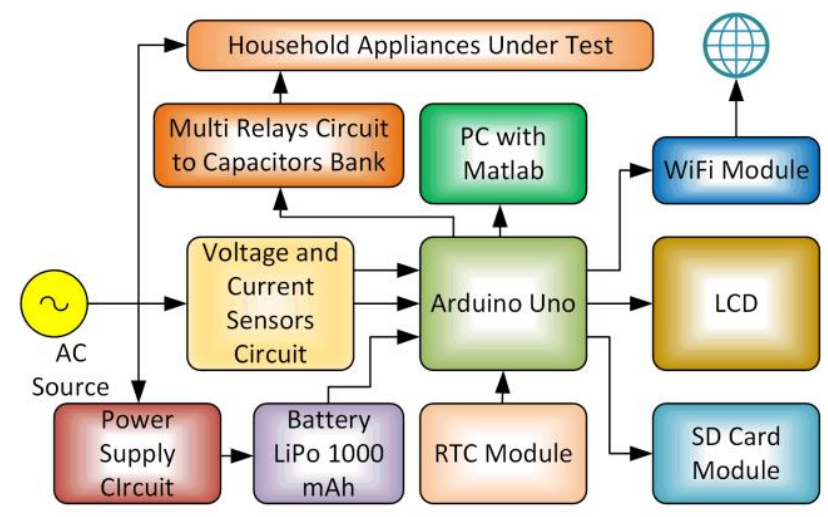

Figure 3. Block diagram of power factor meter using IoT

For the current sensor, there are two types, which is invasive and non-invasive current sensor. The invasive type could provide higher accuracy, while the non-invasive type could provide easier installation. For this reason, we selected a non invasive current sensor, as shown in Figure 4, with proper calibration to improve its accuracy. In addition, the price of the non-invasive AC current sensor is cheaper than the invasive type, thus low cost development of the designed power factor will be achieved. However, an electromagnetic interference could cause measurement error as discussed in [22]. Therefore, care must be taken to use the non-invasive current sensor by wrapping it with aluminum foil to simulate Faraday's cage.

Figure 4 shows the hardware design of the proposed device. An SD card module along with proper software library is used as interface between SD card and Arduino for data logging purposes of the measured real power, apparent power, and power factor along with the date and time stamp obtained from the RTC module. If multiple smart meters are deployed in different locations, then it would be better to use a GPS module which could provide a synchronize date/time and smart meters' location. WiFi module or Ethernet module could be used to provide internet connection to the device for remote monitoring purposes. 


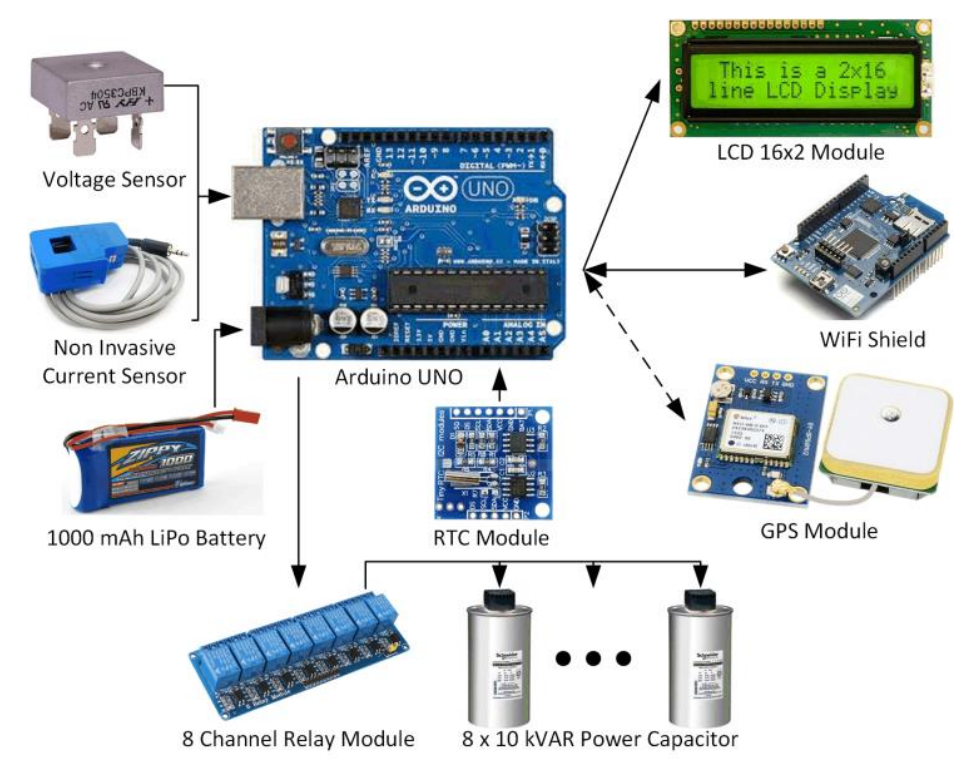

Figure 4. Hardware design of power factor meter using IoT

\subsection{Software Design}

Figure 5 shows the software flowchart of the proposed power factor meter. First, the device is connected to the electrical appliances under test, such as laptop, fan, light bulb, or other electical appliance. Then the voltage and current sensors will measure the voltage and current, respectively. The interfacing circuit will convert into lower voltage and current so that it could be digitized by Arduino's ADC. Next, the real and apparent power will be calculated, in which the power factor could be derived. The measurement result could be displayed at LCD, logged at SD card, or sent to PC for plotting and monitoring in Matlab. Furthermore, if the Wifi or Ethernet module is activated and connected, the result could also be sent to a server for remote monitoring and further analysis. Power factor improvement sub routine will activate the relay module to shunt the capacitor(s) with the electrical appliance.

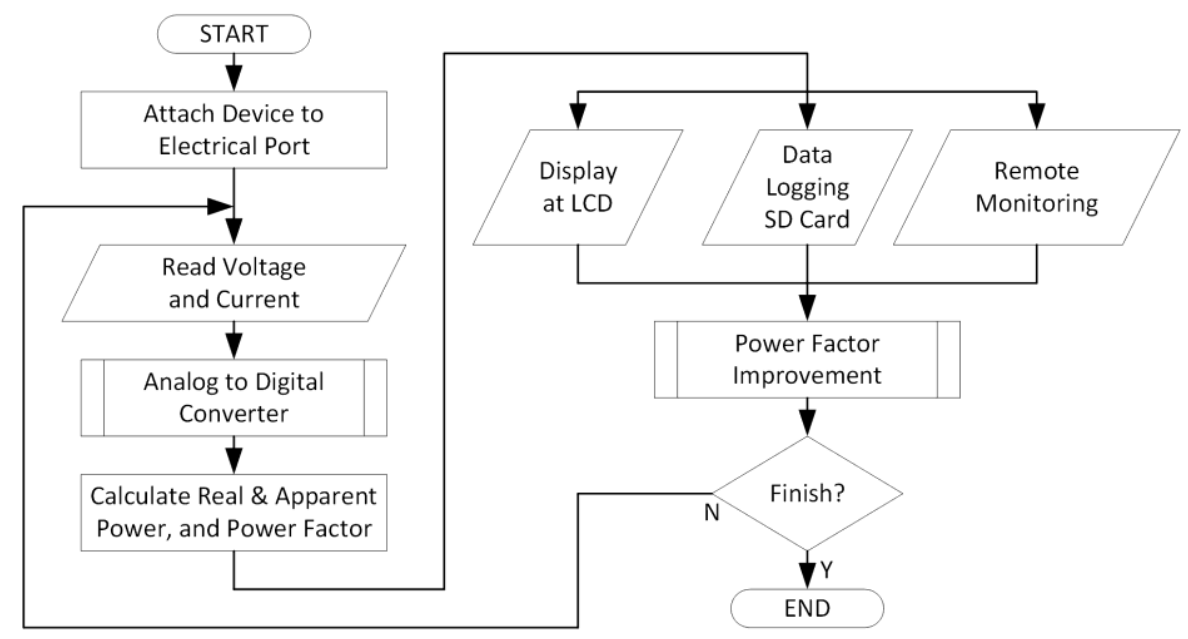

Figure 5. Flowchart of power factor meter and power factor improvement

Automatic power factor correction could be done by employing various capacitors connected in parallel and switched on demand using Arduino output connected to relay circuit. This scenario is shown in Figure 6. The relay circuits which can toogle switch $R_{1}, R_{2}, R_{3}$, (or any $N$ number of capacitors) is connected to the digital output of Arduino. Depends on the current power factor, the proposed device can switch one or more capacitors to improve the power factor. 


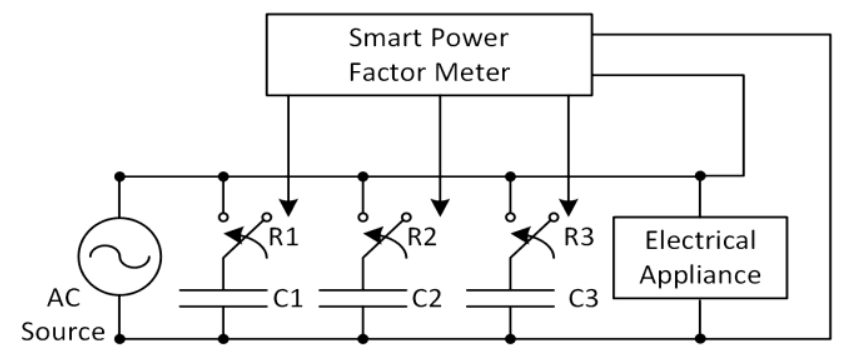

Figure 6. Automatic power factor improvement using capacitor bank

\section{RESULTS AND DISCUSSION}

This section will provide the simulation of power factor correction, calibration of power factor meter, experiments on industrial fan and data logging, as well as applying IoT framework for complete solution.

\subsection{Power Factor Correction Simulation}

Several Matlab/Simulink models were available on various aspects of electrical power simulation [23]. A Matlab program was adopted from [24] to simulate the power factor correction and the required capacitor bank. Figure 7 shows the simulation result which confirm the manual calculation as shown in Figure 2(b). More details simulation on induction motors paremeter, as typical resistive-inductive electrical load, could use the Moto Python toolbox [25].

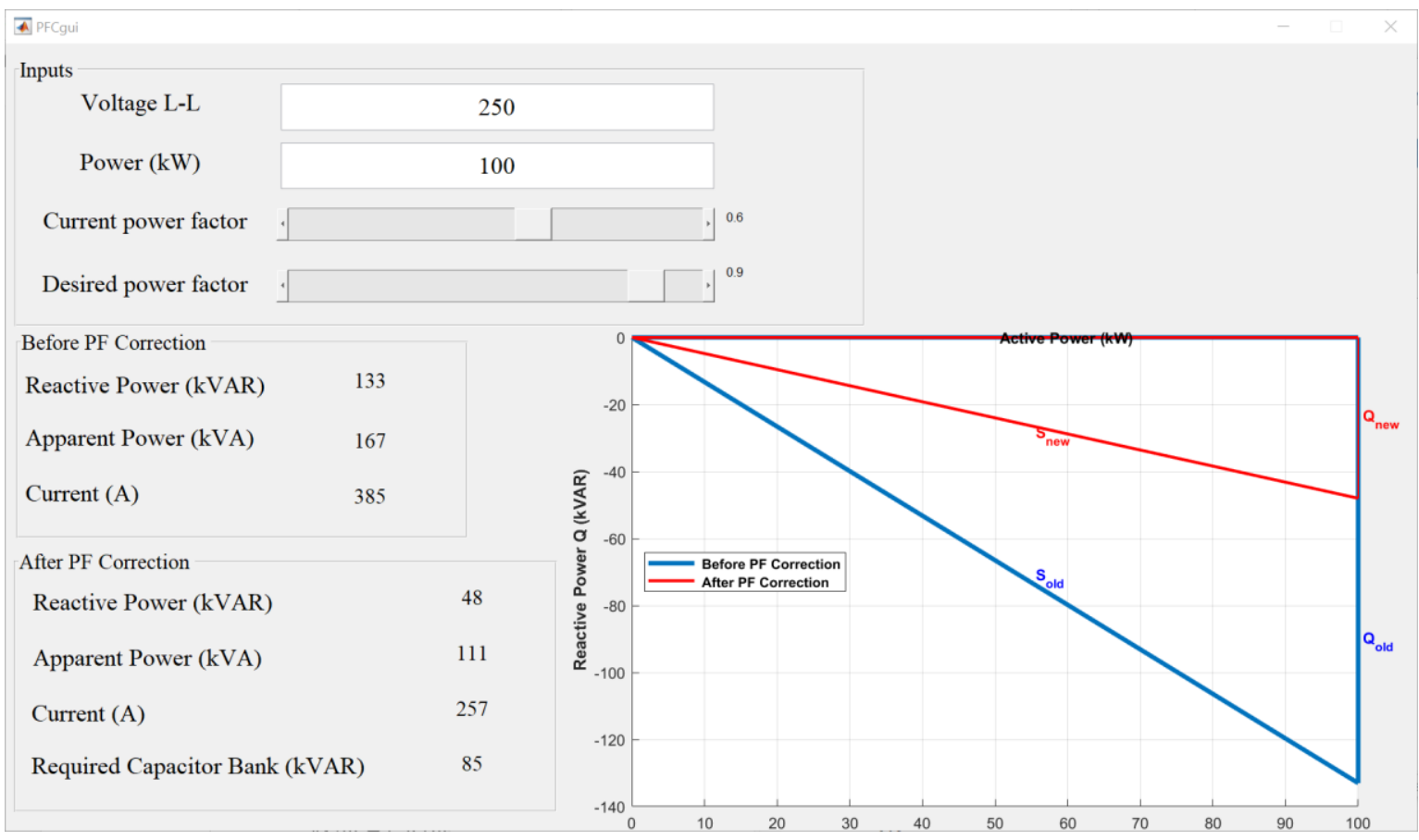

Figure 7. Power factor correction simulation using matlab

\subsection{Calibration}

The use of non-invasive current sensor simplify the implementation while sacrificing its accuracy. The sensor is clipped to the cable which may have different characteristics. Therefore, the calibration process is necessary at least once or when the cable used is changed. There are three devices used to calibrated the proposed power factor meter as shown in Table 1. There are two current measurements, the measurement by non-invasive current sensor, and the measurement by ampere meter. The difference between both measurements will be compensated so that the non-invasive measurement will have the same value 
as the manual ampere meter measurement as shown in Figure 8. After several experiments using three devices, we found that a factor of $\mathbf{0 . 2 3}$ needs to be multiplied to the measured current by non-invasive sensor. Using this correction factor, the difference between the non-invasive current sensor and the ammeter is only around $\pm 1.79 \%$.

Table 1. List of Devices used for Calibration

\begin{tabular}{clcc}
\hline No & \multicolumn{1}{c}{ Devices } & Current (A) & Power (W) \\
\hline 1 & Laptop ASUS Model S200E & 0.17 & 40 \\
2 & Industrial Fan & 0.96 & 230 \\
3 & Light Bulb 100 W & 0.42 & 100 \\
\hline
\end{tabular}

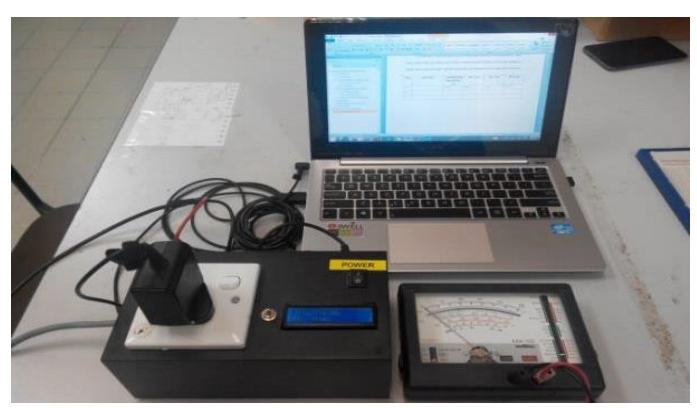

Figure 8. Current measurement calibration process

\subsection{Experiment on Power Factor Measurement on Industrial Fan}

The industrial fan has three speed, i.e. low, medium, and high. Its model number is RIF-650, 26 inch Industrial Fan, with power rating of $230 \mathrm{~W}$. Experiment is conducted to evaluate the measured current and power factor for different fan speed, as shown in Table 2. Based on the experimental results, the best power factor achieved is 0.73 with 0.5 A current, when the speed of the fan is at medium. It can be concluded that the most efficient and lower cost operation for the industrial fan is achieved at medium speed.

Table 2. Current and Power Factor Measurement on Industrial Fan

\begin{tabular}{clcc}
\hline No & Speed & Current $(\mathrm{A})$ & Power Factor \\
\hline 1 & Low & 0.45 & 0.60 \\
2 & Medium & 0.50 & 0.73 \\
3 & High & 0.64 & 0.69 \\
\hline
\end{tabular}

\subsection{Experiment on Data Logging}

For this experiment, the power factor meter is connected with 4 ways extension sockets as shown in Figure 9(a). The experiment is conducted at Robotic Design Laboratory, E2-1, IIUM, for the duration of one day. The power factor data is recorded for about 20 points at various interval in the morning (6.00 to 11.00), afternoon (12.00 to 19.00) and night (20.00 to 5.00), respectively, as shown in Figure 9(b). The data is time series, in which date and time, as well as power factor and current reading were recorded. The data was saved to the SD card. The date and time information were provided by the RTC module. For further processing, the stored data in the SD card was then connected to a PC to be analyzed using Matlab.

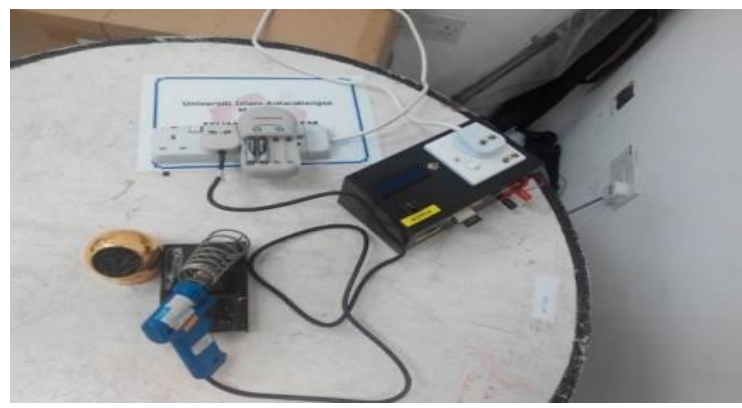

(a) Experimental Setup

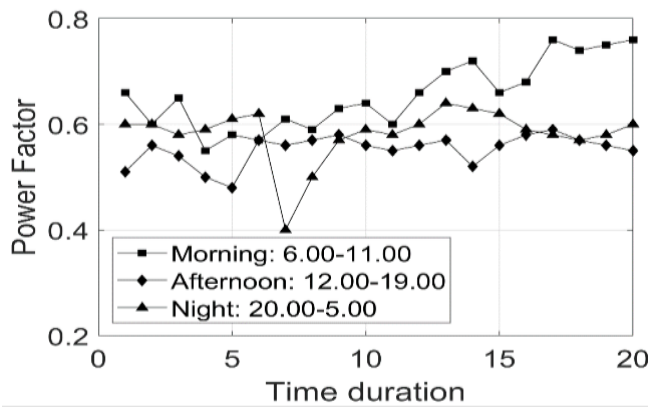

(b) Power Factor Data Recorded

Figure 9. Experiment on Data Logging 
By observing power factor graph as shown in Figure 9(b), it clearly shows that the power factor is fluctuated depends on the usage at that particular time. The average power factor in the morning, afternoon, and night are $0.66,0.55$, and 0.58 , respectively. In the afternoon, many students were doing their Integrated Design Project (IDP), in which equipment usage were hight, including powering the project's device and laptop. While at night, it recorded the worst power factor at 0.4 . This could be due to the lighting and air conditioning were active at that time which brought down the overall power factor in the laboratory.

\subsection{IoT Framework Analysis}

In [7], the IoT Framework, which can be applied for the proposed smart meter, is discussed and divided into several stages, including Things, Connect, Collect, Learn, Do. In our case, the Things is the IoT device and sensors for the smart meter as explained in Figure 3 and 4. For the Connect part, it is not limited to WiFi or Ethernet but it can be other communication technologies, such as Bluetooth, Zigbee, LoRa, etc [21]. The Collect part is the time series databases which could be used to collect the machine information and the power quality information, i.e. current and power factor, with date and time stamp [26, 27]. The Learn part is the machine learning process which could learn the most efficient power factor at particular time using deep learning $[28,29]$. The Do part is the actual implementation of what we have learned from the timeseries database to lower the electricity cost and more efficient electrical appliance operation.

\section{CONCLUSION}

This paper has presented the design of power factor meter using IoT. Using the WiFi or Ethernet module on Arduino, the device could be accessed remotely or sent the data to remote server for data logging and further analysis. Power factor improvement was designed using multi relays connected to capacitor banks with various capacities. To measure the current, a non-invasive current sensor was used for ease of installation. The accuracy of the selected sensor was calibrated using the manual ammeter, in which the difference is around $\pm 1.79 \%$. Finally, the IoT framework analysis was conducted on the proposed device. Further research includes experiments on various electrical appliances, transient effect analysis of automatic power factor correction, and deep learning algorithms applied to the collected time-series database.

\section{ACKNOWLEDGEMENTS}

The authors would like to thank International Islamic University Malaysia (IIUM), Universiti Teknologi MARA (UiTM) Shah Alam and University of New South Wales (UNSW) for providing facilities to support the research work.

\section{REFERENCES}

[1] L. L. Grigsby, Electric power generation, transmission, and distribution, CRC press, 2016.

[2] D. Alahakoon and X. Yu, "Smart electricity meter data intelligence for future energy systems: A survey," IEEE Transactions on Industrial Informatics, vol. 12, pp. 425-436, 2016.

[3] E. Spanò, L. Niccolini, S. Di Pascoli, and G. Iannacconeluca, "Last-meter smart grid embedded in an Internet-ofThings platform," IEEE Transactions on smart grid, vol. 6, pp. 468-476, 2015.

[4] N. K. Suryadevara, S. C. Mukhopadhyay, S. D. T. Kelly, and S. P. S. Gill, "WSN-based smart sensors and actuator for power management in intelligent buildings," IEEE/ASME transactions on mechatronics, vol. 20, pp. 564-571, 2015.

[5] D. Alahakoon and X. Yu, "Advanced analytics for harnessing the power of smart meter big data," in Intelligent Energy Systems (IWIES), 2013 IEEE International Workshop on, pp. 40-45, 2013.

[6] L. Pocero, D. Amaxilatis, G. Mylonas, and I. Chatzigiannakis, "Open source IoT meter devices for smart and energy-efficient school buildings," HardwareX, vol. 1, pp. 54-67, 2017.

[7] T. Chou, Precision-Principles, Practices and Solutions for the Internet of Things, McGraw-Hill Education, 2017.

[8] B. C. Kok, C. Tan, and E. Sulaiman, "Development of a cost-effective op-amp based digital power factor meter," in First Engineering Conference: Energy and Environment (EnCon), 2007.

[9] B. Jovanović, M. Zwolinski, and M. Damnjanović, "Low power digital design in Integrated Power Meter IC," in Proceedings of the Small Systems Simulation Symposium, pp. 49-55, 2010.

[10] S. Rustemli and M. Ates, "Measurement and simulation of power factor using pic16f877," Przegląd Elektrotechniczny (Electrical Review), pp. 0033-2097, 2012.

[11] S.-W. Luan, J.-H. Teng, S.-Y. Chan, and L.-C. Hwang, "Development of a smart power meter for AMI based on ZigBee communication," in Power Electronics and Drive Systems, 2009. PEDS 2009. International Conference on, pp. 661-665, 2009.

[12] T. S. Gunawan, M. H. Anuar, M. Kartiwi, and Z. Janin, "Development of Power Factor Meter using Arduino," in 2018 IEEE 5th International Conference on Smart Instrumentation, Measurement and Application (ICSIMA), pp. $1-4,2018$. 
[13] I. E. Commission, "IEC 61000 series: Electromagnetic Compatibility," 2018.

[14] IEEE, "IEEE Standard 519, IEEE recommended practices and requirements for harmonic control in electric power systems," 1992.

[15] S. Halpin, "Comparison of IEEE and IEC harmonic standards," in IEEE Power Engineering Society General Meeting, 2005, pp. 2214-2216, 2005.

[16] IEEE, "IEEE Guide for Application of Shunt Poewr Capacitors," 2010.

[17] F. Zheng and W. Zhang, "Long term effect of power factor correction on the industrial load: A case study," in 2017 Australasian Universities Power Engineering Conference (AUPEC), pp. 1-5, 2017.

[18] M. A. Masoum and E. Fuchs, Power Quality in Power Systems and Electrical Machines, 2nd Edition, Elsevier, 2015.

[19] A. Cano Ortega, F. J. Sánchez Sutil, and J. De la Casa Hernández, "Power Factor Compensation Using Teaching Learning Based Optimization and Monitoring System by Cloud Data Logger," Sensors, vol. 19, pp. 2172, 2019.

[20] T. S. Gunawan, I. R. H. Yaldi, M. Kartiwi, and H. Mansor, "Performance Evaluation of Smart Home System using Internet of Things," International Journal of Electrical and Computer Engineering, vol. 8, pp. 400-411, 2018.

[21] T. S. Gunawan, I. Rahmithul, H. Yaldi, M. Kartiwi, and N. Ismail, "Prototype design of smart home system using internet of things," Indonesian Journal of Electrical Engineering and Computer Science, vol. 7, pp. 107-115, 2017.

[22] F. Leferink, C. Keyer, and A. Melentjev, "Static energy meter errors caused by conducted electromagnetic interference," IEEE electromagnetic compatibility magazine, vol. 5, pp. 49-55, 2016.

[23] E. P. Leite, Matlab-modelling, programming and simulations, ABM Nasiruzzaman, 2010.

[24] M. Musleh, "Power Factor Correction," Mathworks, May 25, 2017. [Online]. Available: https://www.mathworks.com/matlabcentral/fileexchange/63117-powerfactorcorrection, [Accessed Jul 2019].

[25] J. Susanto, "Moto: Induction motor parameter estimation tool," Github, Dec 2, 2017. [Online]. Available: https://github.com/susantoj/Moto, [Accessed Jul 2019].

[26] M. Arlitt, M. Marwah, G. Bellala, A. Shah, J. Healey, and B. Vandiver, "Iotabench: an internet of things analytics benchmark," in Proceedings of the 6th ACM/SPEC International Conference on Performance Engineering, pp. 133-144, 2015.

[27] B. Leighton, S. J. Cox, N. J. Car, M. P. Stenson, J. Vleeshouwer, and J. Hodge, "A best of both worlds approach to complex, efficient, time series data delivery," in International Symposium on Environmental Software Systems, pp. 371-379, 2015.

[28] H. Shi, M. Xu, and R. J. I. T. o. S. G. Li, "Deep learning for household load forecasting-A novel pooling deep RNN," IEEE Transactions on Smart Grid, vol. 9, pp. 5271-5280, 2017.

[29] Y. Wang, Q. Chen, D. Gan, J. Yang, D. S. Kirschen, and C. J. I. T. o. S. G. Kang, "Deep learning-based sociodemographic information identification from smart meter data," IEEE Transactions on Smart Grid, vol. 10, pp. 2593-2602, 2018.

\section{BIOGRAPHIES OF AUTHORS}

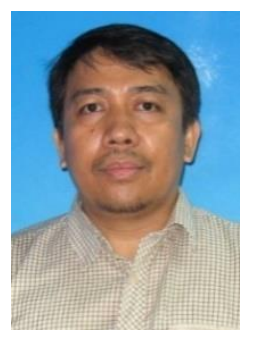

Teddy Surya Gunawan received his B.Eng degree in Electrical Engineering with cum laude award from Institut Teknologi Bandung (ITB), Indonesia in 1998. He obtained his M.Eng degree in 2001 from the School of Computer Engineering at Nanyang Technological University, Singapore, and $\mathrm{PhD}$ degree in 2007 from the School of Electrical Engineering and Telecommunications, The University of New South Wales, Australia. His research interests are in speech and audio processing, biomedical signal processing and instrumentation, image and video processing, parallel computing, and IoT. He is currently an IEEE Senior Member (since 2012), was chairman of IEEE Instrumentation and Measurement Society - Malaysia Section (2013 and 2014), Associate Professor (since 2012), Head of Department (2015-2016) at Department of Electrical and Computer Engineering, and Head of Programme Accreditation and Quality Assurance for Faculty of Engineering (2017-2018), International Islamic University Malaysia. He is Chartered Engineer (IET, UK) and Insinyur Profesional Madya (PII, Indonesia) since 2016, and registered ASEAN engineer since 2018.

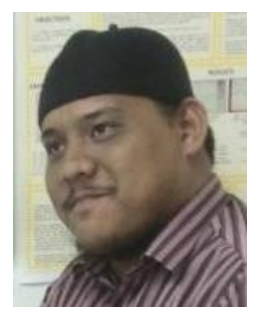

Muhamad Hadzir Anuar has completed his B.Eng. (Hons) degree in Communication Engineering, International Islamic University Malaysia (IIUM) in 2014 and is currently working at Sony EMCS (Malaysia) since 2014 as software design engineer and then as software quality assurance (SQA) engineer. His research interests are in software engineering, instrumentation and signal processing. 


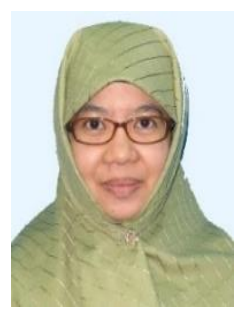

Mira Kartiwi completed her studies at the University of Wollongong, Australia resulting in the following degrees being conferred: Bachelor of Commerce in Business Information Systems, Master in Information Systems in 2001 and her Doctor of Philosophy in 2009. She is currently an Associate Professor in Department of Information Systems, Kulliyyah of Information and Communication Technology, and Deputy Director of e-learning at Centre for Professional Development, International Islamic University Malaysia. Her research interests include electronic commerce, data mining, e-health and mobile applications development.

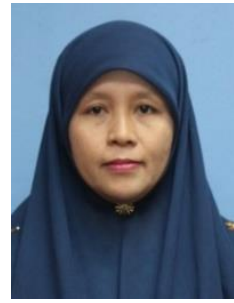

Zuriati Janin received her B.Eng in Electrical Engineering from the Universiti Teknologi Mara, Malaysia in 1996 and MSc. in Remote Sensing \& GIS from the Universiti Putra Malaysia (UPM) in 2001. In 2007, she began her study towards a Ph.D in Instrumentation and Control System at the Universiti Teknologi Mara, Malaysia. She has served as a lecturer at Universiti Teknologi Mara for more than 20 years and currently she is a Senior Lecturer at Faculty of Electrical Engineering, UiTM, Shah Alam. She has been involved with IEEE since 2012 and been mainly working with IEEE Instrumentation \& Measurement Chapter (IM09), Malaysia Section since 2013. The IM09 acknowledged her role as a founder Treasurer in initiating and promoting ICSIMA as a series of annual chapter's flagship conferences since its inception in 2013. She also has more than 10 years experiences in organizing the International Conferences, Workshops and Seminars. Her role as a conference treasurer started since 2005. 